\title{
Proposição de uma escala de avaliação da qualidade de parques públicos verdes urbanos
}

Proposition of a scale for evaluating the quality of urban green public parks

Ítalo Brener Carvalho

Professor Adjunto no Centro Federal de Educação Tecnológica de Minas Gerais - CEFETMG, Belo Horizonte/MG, Brasil

E-mail: italobrener@hotmail.com

Marlusa Gosling

Professora Associada de Marketing da Universidade Federal de Minas Gerais - UFMG, Belo Horizonte/MG, Brasil

E-mail: mg@gmail.com.br 


\section{RESUMO}

Áreas verdes urbanas são comumente avaliadas por duas perspectivas: a primeira aponta dimensões relacionadas com benefícios para os indivíduos e consequentemente para a população local e na segunda perspectiva relacionada com a atratividade turística com dimensões focadas em atrair investimento e renda para a população e para a cidade. Por meio de um aporte teórico fundamentado em marketing de serviços, este estudo propõe um avanço das escalas existentes como, por exemplo, a SERVIQUAL, SERVICESCAPE e a IQAVU. Com o objetivo de desenvolver uma escala para a avaliação da qualidade percebida pelos visitantes de parques verdes urbanos, na cidade de Belo Horizonte, MG - Brasil. Este trabalho aponta indicadores de qualidade em áreas verdes urbanas sinalizados por usuários locais e/ou por turistas. Para alcançar o objetivo foi realizada uma revisão bibliográfica, a 17 vistas sistemáticas aos parques na cidade de Belo Horizonte. Os dados obtidos, de caráter exploratório foram analisados a partir da técnica de análise de conteúdo, atingiram a saturação ao numero de 77 entrevistas e forneceram o subsidio necessário para a elaboração de um segundo método. Caracterizado por um estudo do tipo survey, com uma coleta de 737 questionários estruturados, analisados por Analise Fatorial Exploratórios (AFE) para confirmar que a qualidade percebida é um construto multidimensional, composto por (1) Aspectos Tangíveis, (2) Facilidade Avaliações de Acesso, (3) Avaliação Segurança, (4) Avaliação Beleza, (5) Avaliação Propensão a Integração. A escala proposta é adequada para a avaliação da qualidade dos espaços verdes, contribuindo para ações que visem maior motivação de visita e satisfação de seus usuários.

Palavras-chave: Qualidade Percebida. Satisfação. Atitudes e Intenções. Comportamentais. Modelagem de Equações. Áreas Verdes Urbanas.

\section{ABSTRACT}

Urban green areas are commonly evaluated by two perspectives: the first points to dimensions related to benefits for the individuals and consequently for the local population and in the second perspective, tourist have dimensions focused on attracting investment and income for the population and the city. Through a theoretical contribution based on service marketing, this study proposes an advance of existing scales such as SERVIQUAL and SERVICESCAPE and IQAVU. The objective of this work is to develop a scale for the evaluation of the total quality perceived by the visitors of urban green parks, in the city of Belo Horizonte, MG - Brazil. To reach the objective, the following methodological phases were followed: A bibliographical review and 17 systematic views of the parks in the city of Belo Horizonte. The exploratory data were analyzed using the content analysis technique, reached saturation at the number of 77 interviews and provided the necessary subsidy for the elaboration of a second method. Characterized by a survey-type study, with a collection of 737 structured questionnaires, analyzed by Exploratory Analysis (AFE) to confirm that the perceived quality is a multidimensional construct, composed of (1) Tangible Aspects, (2) Access Assessment Facility, (3) Security Assessment, (4) Beauty Assessment, (5) Propensity Assessment, Integration. The proposed scale is adequate for the evaluation of the quality of green spaces, contributing to actions that aim at greater motivation to visit and satisfaction of its users.

Keywords: Perceived Quality. Satisfaction. Attitudes and Intentions. Behavioral. Modeling of Equations. Urban Green Areas. 


\section{INTRODUÇÃO}

A gestão do espaço público intermediada pelas relações presentes entre cidade e o espaço público representa um campo de investigação importante, na atualidade, evocada pelo ambiente urbano (Castilho, Dolbec, \& Veresiu, 2016). Espaços públicos de qualidade refletem a qualidade de vida, projetam uma cidade, atraem turistas e geram bem-estar (Sempik \& Bragg, 2013). A dinamicidade e a subjetividade para a avaliação destes espaços conduzem a uma constante necessidade de estabelecer critérios, indicadores e metodologias capazes de, corretamente, avaliar generalidades e serviços (Ostrom, Parasuraman, Bowen, Patricio, \& Voss, 2015), sugerindo novas pesquisas em diferentes contextos (Parasuraman, Zeithaml, \& Berry, 1985).

Sendo, portanto um desafio cada vez maior gerir e planejar a demanda por uma cidade mais eficiente, justa e habitável, na qual a interação entre os cidadãos e a apropriação do espaço urbano contribua para uma maior qualidade de vida (Davis, Ozanne, \& Hill, 2016).

Segundo Bragg, Wood, Barton e Pretty (2015) o acesso a áreas verdes contribuem para o bem-estar físico e mental, espaços públicos de lazer e recreação relacionam-se com a equidade social e reduzem conflitos de natureza social. Em contextos geográficos ambientais, econômicos e culturais, a distribuição justa destes locais faz parte do processo de urbanização (Wendel, Zargerb, \& Mihelcic, 2012) e de efeitos positivos relacionados à saúde física e saúde mental de seus habitantes (Davis, Ozanne, \& Hill, 2016).

Porém mensurar a qualidade percebida pelos usuários de parques não está, segundo Edginton e Chen (2014), centrada apenas ao bem viver físico e mental. Por meio do consumo de lazer os autores apontam ainda novas centralidades como a obtenção de maior conhecimento, o desenvolvimento de habilidades e de valores edificantes em uma sociedade (Crilley, Weber, \& Taplin, 2012) além de transformarem o indivíduo (Edginton \& Chen, 2014).

Dessa forma ao se acompanhar a satisfação dos usuários com os serviços prestados em espaços de lazer urbanos auxiliariam, principalmente na solução prática em dois momentos: (1) verificar o que pode está em desacordo entre a gestão dos parques e o usuário e (2) predizer o que seria necessário para o futuro, quais mudanças ao longo prazo precisariam ser repensadas pela administração pública naquele espaço urbano (Crilley, Weber, \& Taplin, 2012).

Contribuindo assim em ampliar a utilização de equipamentos públicos de lazer, a partir das necessidades e demandas dos usuários (Sterry, 2004). E em especifico na avaliação 
da qualidade dos serviços prestados em paisagens verdes na cidade de Belo Horizonte (Costa, Álvares, Maciel, Teixeira, Coimbra, Simão, Perna, \& Godinho, 2009).

A avaliação por parte dos visitantes de espaços verdes perpassa, portanto, por atributos já apontados como a investigação do serviço prestado, pela percepção e pela experiência do usuário, pela qualidade percebida e pelas dimensões da qualidade de serviços (Parasuraman, Zeithaml, \& Berry, 1985). E a frequência de visitação depende da percepção da qualidade em relação ao parque visitado (Kim, Lee, \& Klenosky, 2003) das suas motivações (Crilley, Weber, \& Taplin, 2012) e do sentimento de bem-estar (Bragg, Wood, Barton, \& Pretty, 2015) do usuário.

O objetivo geral deste artigo é avaliar pela perspectiva das dimensões da qualidade: Qual a avaliação que os usuários fazem de parques verdes na cidade de Belo Horizonte. E ainda mais especificamente buscar-se-á: (a) Identificar qualitativamente quais são os atributos de qualidade percebidos pelos usuários de áreas verdes; (b) Avaliar a percepção da qualidade de usuários de parques em relação aos serviços oferecidos por este equipamento em Belo Horizonte, MG; (c) Identificar a satisfação dos usuários/visitantes em relação a este espaço de lazer e recreação; (d) Identificar as atitudes e intenções comportamentais de usuários com ênfase para os seguintes construtos: aspectos tangíveis, acesso, acessibilidade interna, segurança, estética, conforto ao usuário e sociabilidade.

\section{MARKETING DE SERVIÇOS}

A relevância do setor de serviços se reflete no grande numero de pesquisas realizadas neste campo, influenciadas principalmente pela relevância econômica segundo Lovelock, Wirtz e Hemzo (2011). A oferta de serviços de saúde, de educação ou de transporte, por exemplo, são consideradas fundamentais e essenciais como políticas sociais (Hoffman \& Bateson, 2003). Porém, os serviços de lazer, muitas vezes são deixados com menos investimentos e projetos são descontinuados. Segundo Barra, Silva, Miranda, \& Vitorino (2014), a estruturação e implantação de sistemas avaliatórios voltados a políticas públicas do esporte e lazer ainda dão seus primeiros passos na batalha pela busca de recursos públicos.

Segundo Lovelock, Wirtz e Hemzo (2011) investigar como os consumidores utilizam ou avaliam serviços é uma tendência. Segundo Hoffman e Bateson (2003), mesmo em organizações sem fins lucrativos (ou em serviços sociais) o desafio de ampliar seu público, de gerar visitas mais frequentes, e ampliando o acesso (Saatcioglu \& Ozanne, 2013), de 
levantamento de fundos para melhoria de suas instalações e de melhoria ou de novos serviços é um desafio (Wendel, Zargerb, \& Mihelcic, 2012).

\section{UMA LÓGICA DOMINADA PELO SERVIÇO}

Como destacado pelas pesquisas de Zeithaml, Parasuraman e Berry (1990), de Hoffman e Bateson (2003), de Lovelock, Wirtz e Hemzo (2011) e de Akaka, Corsaro, Kelleher, Maglio, Seo, Lusch, e Vargo, (2014) a potencialidade e a relevância social e econômica do setor de serviço é amplamente discutida. Impulsionados por um questionamento de uma lógica predominantemente voltada para produtos, destaca a proposta de uma lógica que considere também uma ótica voltada para o conceito de serviço, Vargo e Lusch (2004) introduziram o conceito da Service-Dominant Logic ou SD-L.

Para Prahalad e Ramaswany (2004), a Lógica Dominada pelo Serviço rompe modelos tradicionais centrados no produto, centralizado na definição de valor pela firma, alterada para uma ótica de valor determinado pelo consumidor. A possibilidade de se conhecer melhor e de perto o comportamento do consumidor, possibilitaria uma prestação de um serviço mais adequado (Blackwell, Miniard, \& Engel, 2013).

A SD-L incorpora as características do serviço (inseparabilidade, intangibilidade, variabilidade ou heterogeneidade e perecibilidade) explorado assim o conceito do valor em uso sendo, segundo Vargo e Lusch (2004) avaliados pelos consumidores durante o uso de um serviço. Segundo Bettencourt, Lusch e Vargo (2014), a contribuição desta perspectiva está na transferência de uma noção de valor transferida ao cliente durante uma compra. Uma noção de valor que é criada conjuntamente com clientes no contexto do uso (Prahalad \& Ramaswany, 2004) no caso de serviços.

Vargo e Lusch (2016) propõem pensar as características dos serviços como entidades dinâmicas disponíveis para o uso. Envolvem a interação entre o beneficiário, a natureza do serviço e a cultura, criando um ecossistema de serviços (Akaka, et al., 2014) e de cocriação de valores entre seus atores (Tombs \& McColl-Kennedy, 2013).

O termo ecossistema é escolhido por Wieland, Polese, Vargo e Lusch (2012), por indicar uma dinâmica de adaptação auto ajustáveis, simultaneamente reconfigurando o processo de integração de recursos e criação de valor constante do sistema. Já a cocriaçao de valor seria contextualmente: experiencial (Verhoef, Lemon, Parasuraman, Roggeveen, Tsiros, \& Schlesinger, 2009). Isso significa que criação de valor é interaciona (Prahalad \& Ramaswany, 2004), os atores consumidores, os beneficiários do serviço de forma individualizada percebem no ato experiencial de consumo se ganham ou perdem nessa 
proposição de valor (Vargo \& Lusch, 2008). Já que a qualidade percebida pelo usuário inclui o entendimento de como os serviços e clientes interagem, colocando os próprios usuários como fonte de avaliação, de colaboração e de melhoria do serviço (Verhoef, et al., 2009).

\section{DETERMINANTES E LACUNAS DA QUALIDADE EM SERVIÇOS}

Já apontando por Zeithaml, Parasuraman e Berry (1990) os serviços apresentam uma desvantagem clara em relação aos bens físicos, uma vez que a sua avaliação de qualidade envolve fatores de mensurabilidade intangíveis. Da mesma forma, estas características da qualidade no serviço prestado por uma área verde também não são facilmente percebidas pelos usuários. Segundo Lindon, Lendrevie, Lévy, Dionísio e Vicente (2013), o caráter intangível dos serviços, não tem uma componente física, material, que permita observação direta e análise prévia.

Isso significa que os serviços, em geral, possuem características bastante distintas daquelas observadas nos bens físicos (Gronroos, 2009) tornando a avaliação da qualidade em serviço, de certa forma, subjetiva. Sendo, portanto necessário a apreciação de variáveis de mensuração e ferramentas capazes de medir percepções com razoável grau de objetividade (Kaplan \& Norton, 2003).

Elencadas por Parasuraman, Zeithaml e Berry (1985) e Zeithaml, Parasuraman e Berry (1990) as características únicas dos serviços: a intangibilidade, heterogeneidade, inseparabilidade e perecibilidade dificultam o constante aperfeiçoamento na mensuração dos padrões de qualidade dos serviços (como os que são oferecidos em parques verdes urbanos). Esta complexidade em mensurar a qualidade de serviços, acontece pelas características inerentes ao serviço.

A primeira delas, considerada a principal das diferenças, é a intangibilidade. Hoffman e Bateson (2003) afirmam que diferentemente de quando se adquiri um bem e o consumidor se apodera fisicamente do produto, em serviços ao contrário, o que se tem é uma experiência. O mesmo autor afirma ainda que a experiência não pode ser embrulhada, carregada para outro lugar, ficando apenas na memória do indivíduo.

A segunda, a perecibilidade, consiste na incapacidade de estocagem e armazenamento, ou seja, uma vez ofertado, o serviço está disponível, caso não sejam usufruídos ou consumidos, não podem ser revendidos ou consumidos no futuro (Parasuraman \& Berry, 1990). A terceira é a inseparabilidade, faz com que os serviços sejam produzidos na presença do consumidor, exigindo, muitas vezes, a participação ou o comprometimento dele. Esta 
participação do consumidor diz respeito às ações e aos recursos fornecidos por ele durante a entrega de serviços e inclui insumos materiais, físicos e até emocionais (Lovelock, Wirtz, \& Hemzo, 2011). Esta característica refere-se ao papel do consumidor como principal agente criador de valor durante o consumo de um serviço.

A presença da heterogeneidade, como quarta característica do serviço refere-se as diferenças que se baseiam nas caraterísticas determinantes, nas variações na prestação dos serviços, o que torna difícil reparar algum erro sem que já tenha sido seja percebido. No modelo original de Zeithaml, Parasuraman e Berry (1990) variabilidade ou heterogeneidade e suas implicações gerenciais fundamentais de serem reconhecidas.

Modelo de lacunas na qualidade de serviço Parasuraman, Zeithaml e Berry, (1985) Zeithaml e Bitner (2003) originalmente apresentam 4 lacunas e uma lacuna do cliente, o modelo proposto apresenta as seguintes lacunas.

Figura 1 - Modelo lacunas da Qualidade dos Serviços

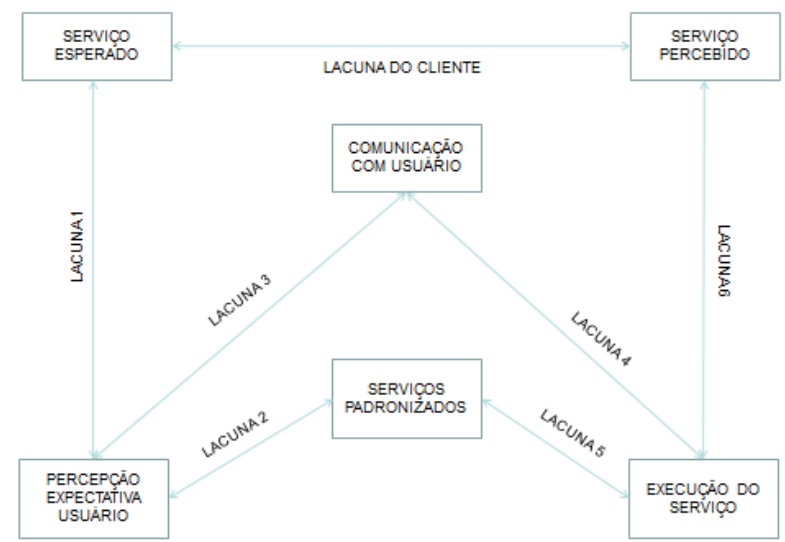

Fonte: Parasuraman, Zeithaml e Berry, 1985; Zeithaml e Bitner, 2003.

1. Lacuna 1: é a diferença entre as percepções dos gestores sobre as expectativas dos clientes e as expectativas que o usuário possui.

2. Lacuna 2: é a diferença entre a percepção dos gestores das expectativas dos usuário em confronto com os serviços padronizados.

3. Lacuna 3 e 4: que demonstra a diferença entre o serviço prestado e as comunicações externas do fornecedor ao cliente.

4. Lacuna 5: ou seja, a diferença entre as especificações da qualidade do serviço e a entrega real do serviço.

5. Lacuna 6: pois apresenta a diferença entre as expectativas do Cliente e a percepção dos Gerentes sobre as necessidades e desejos do Cliente.

6. Lacuna do cliente: compreende a percepção entre a combinação de todas as lacunas, resumida pela diferença global entre o serviço esperado e o serviço percebido, medido pela experiência do usuário. 
Como uma estratégia geral, os determinantes da qualidade em serviços, neste caso, podem ser reduzidos, a fim de reduzir as lacunas uma empresa deve: (1) Saber o que os usuários esperam; (2) Estabelecer os padrões corretos para a qualidade do serviço; (3) Garantir que o desempenho do serviço atenda aos padrões; (4) Assegurar que a entrega corresponde às promessas feitas.

Lovelock, Wirtz e Hemzo (2011), ampliam e completam o modelo original de Zeithaml, Parasuraman e Berry (1990) e as suas implicações gerenciais: (a) A maioria dos serviços não pode ser estocada; (b) Elementos intangíveis geralmente dominam a criação de valor; (c) Serviços são normalmente difíceis de visualizar e compreender; (d) Clientes podem ser envolvidos na coprodução; (e) as pessoas podem fazer parte da experiência do serviço; (f) insumos operacionais tendem a variar; (g) tempo é um fator que assume vital importância. Contribuindo como alternativa bem-sucedida para a compreensão da diferenciação entre bens e serviços.

Para Zeithaml, Parasuraman e Berry (1990), os principais aspectos considerados pelos consumidores em relação à qualidade do serviço são tangíveis, confiabilidade, responsividade, segurança e empatia.

Quadro 1 - Dimensões da Qualidade

\begin{tabular}{|c|l|}
\hline Tangíveis & $\begin{array}{l}\text { Referindo-se a aspectos das facilidades físicas, dos equipamentos disponíveis, recursos humanos } \\
\text { e de comunicação. Aparência das instalações físicas, equipamentos, funcionários e material de } \\
\text { comunicação; }\end{array}$ \\
\hline Confiança & $\begin{array}{l}\text { Corresponde à habilidade de organização para desenvolver entregar os serviços prometidos } \\
\text { com confiança e precisão. Capacidade de executar o serviço prometido de forma confiável e } \\
\text { com precisão; determinar a estabilidade e qualidade dos dados obtidos; }\end{array}$ \\
\hline $\begin{array}{c}\text { Presteza } \\
\text { Atendimento }\end{array}$ & $\begin{array}{l}\text { Seria a boa vontade em ajudar usuários e prover um serviço rápido. boa vontade e prontidão em } \\
\text { atender os clientes; }\end{array}$ \\
\hline $\begin{array}{c}\text { Garantia } \\
\text { Segurança }\end{array}$ & $\begin{array}{l}\text { As habilidades que inspirarem confiança e segurança (incluindo competência, cortesia, } \\
\text { credibilidade e segurança). Conhecimento e cortesia e dos funcionários e habilidade de } \\
\text { transmitir confiança e segurança; }\end{array}$ \\
\hline Empatia & $\begin{array}{l}\text { Características correspondentes à atenção, ao cuidado, de forma individualizada que se oferece o } \\
\text { serviço(incluindo aproximação, comunicação e compreensão do cliente). tratar e prover atenção } \\
\text { individualizada aos consumidores. }\end{array}$ \\
\hline
\end{tabular}

Fonte: Zeithaml, Parasuraman e Berry, 1990. 


\section{OS 7PS DO SERVIÇO}

Atualmente, os gestores de parques e agentes públicos encarregados de promover serviços de lazer devem de certa forma, compreender e atentarem para as necessidades de atrair usuários (Vignati, 2008) e justificar o investimento público ou captar recursos para a manutenção destes espaços (Sempik \& Bragg, 2013).

Se satisfação é o resultado que se pretende alcançar o composto mercadológico de serviço os "Sete P's", ou seja, produto, preço, comunicação e distribuição são o meio que contribuem estrategicamente para a intenção de criar um relacionamento com o usuário baseado em atender suas expectativas e aumentar sua frequência, sendo possível angariar subsídios que auxiliariam a conseguir uma melhor satisfação do serviço utilizado. Consequentemente uma avaliação de valor mais elevada por parte do público alvo, que por sua vez gera atividades positivas e maior disposição para interações, satisfação e reconhecimento do serviço prestado por parques (Crilley, Weber, \& Taplin, 2012).

Quadro 2 - Dimensões do composto mercadológico de serviços

\begin{tabular}{|c|l|}
\hline Produto & $\begin{array}{l}\text { No caso de serviços, o 'produto' é intangível, heterogêneo e perecível. Além disso, a sua } \\
\text { produção e consumo são inseparáveis. }\end{array}$ \\
\hline Preço & $\begin{array}{l}\text { O preço dos serviços é mais difícil do que o preço dos produtos. Preço social e } \\
\text { psicológico. }\end{array}$ \\
\hline Praça & $\begin{array}{l}\text { Local onde o comportamento desejado possa ser praticado, interações, tornar o local } \\
\text { conveniente e acessível. }\end{array}$ \\
\hline Promoção & Comunicações de Incentivo do uso do serviço, Símbolos, Criar mensagens apropriadas. \\
\hline Processo & $\begin{array}{l}\text { Confiança, conhecimento, cortesia e educação dos funcionários e suas habilidades em } \\
\text { inspirar crédito e confiança. Treinamento e acompanhamento de pessoal, Empatia } \\
\text { interesse, cuidado, preocupação e atenção. }\end{array}$ \\
\hline $\begin{array}{c}\text { Padrão de serviço é repetidamente entregues aos clientes. Influenciado pelo modo } \\
\text { como ele recebe o serviço e como ele experimenta o processo. }\end{array}$ \\
Física & $\begin{array}{l}\text { Como os serviços são intangíveis em sua natureza, incorporar elementos em sua oferta } \\
\text { para melhorar a experiência do cliente, envolvem, por exemplo: sinalização adequada, } \\
\text { condições do ambiente (limpeza e organização) e a conduta de outros clientes que } \\
\text { estejam utilizando o serviço (por exemplo, o barulho). }\end{array}$ \\
\hline
\end{tabular}

Fonte: Lovelock, Wirtz e Hemzo, 2011.

\section{DIMENSÕES DA QUALIDADE PERCEBIDA}

Aquilo que não pode ser medido, não pode ser gerenciado (Kaplan \& Norton, 1997). Assim como satisfação, a qualidade percebida também é difícil de ser mensurada. Não é fácil medir a satisfação dos clientes, já que esta é determinada por diversos fatores, inclusive fatores intangíveis (Fitzsimmons \& Fitzsimmons, 2000).

Três modelos são constantemente replicados como modelos ideais de avaliação da qualidade em serviços: o SERVQUAL (Zeithaml \& Berry, 1988) e avanços com da 
SERVICESCAPE na intenção comportamental (Chang, 2016), além da proposta de aplicação em áreas verdes de Pereira, Gimenes, Silva e Zanatta (2011) de um indicador de qualidade de áreas verdes urbanas - IQAVU.

As três escalas auxiliam a compreensão das múltiplas facetas ou dimensões que avaliam os serviços, algumas críticas ao modelo SERVQUAL, já foram colocadas segundo Augustyn e Seakhoa-King (2004) a escala pode não ser adequada para medir serviços de lazer, turismo e hospitalidade. E ao SERVICESCAPE por se entender como demasiado complexos e abrangentes, as inúmeras variedade de serviços incluídos, resumidos por Bitner (1992) como o impacto do ambiente verde nos consumidores (Pereira, Gimenes, Silva, \& Zanatta, 2011) levaria apenas a comportamentos de aproximação ou rejeição.

Apesar de suas dimensões serem pertinentes, os critérios utilizados pelos usuários para avaliar a qualidade em serviços, segundo Zeithaml, Parasuraman e Berry (1990), são resumidos pelas cinco dimensões. Os próprios autores da escala Zeithaml e Berry (1988) afirmam que ela pode ser modificada e adaptada a determinadas caraterísticas concretas de uma investigação.

Geralmente a qualidade de serviço é compreendida pela diferença entre a qualidade esperada pelos consumidores (usuários) e aquela oferecida pelo serviço (Parasuraman, Zeithaml, \& Berry, 1988). Dai que precisamente a necessidade de se completar o modelo por uma fase exploratória, que utilize do modelo conceitual, mudando e adaptando as questões de conteúdo, pois existe uma especificidade em cada caso concreto que se pretende aferir.

\section{SATISFAÇÃO EM PARQUES VERDES URBANOS}

A satisfação pode ser definida como uma avaliação atitudinal, que se segue a uma experiência de consumo (Lovelock, Wirtz, \& Hemzo, 2011), isto significaria que os consumidores teriam certos padrões em mente, certas expectativas, construídas tanto por com comentários de outros usuários, como por informações que influenciam no seu processo de escolha.

Uma definição objetiva de satisfação foi proposta por Oliver (1997), de que satisfação é um julgamento resultante, uma confirmação positiva, se o serviço foi melhor do que o esperado ou, se não, confirmação negativa, se foi pior do que o esperado. A comparação entre o que era esperado com o desempenho de um serviço. Na visão de Grönroos (2009) uma boa qualidade percebida é obtida quando a qualidade experimentada atende às expectativas do cliente, isto é, à qualidade esperada. 
Mas segundo Lovelock, Wirtz e Hemzo (2011) a comparação entre expectativa e desempenho nas situações em que consumidores não têm uma escolha livre (em grande parte dos serviços públicos é esta a situação que se aplica) existe o risco em se avaliar satisfação pela expectativa previa nestas situações, seria melhor, usar na comparação, a relação entre o atendimento das necessidades ou desejos (Ostrom, Parasuraman, Bowen, Patricio, \& Voss, 2015).

Em avaliações especificas de parques urbanos alguns dois fatores segundo Christopher, Payne e Ballantyne (1991) evidenciam um usuário satisfeito: (a) a intenção de retornar e (b) a intenção de recomendar. Já (White, Pahl, Ashbullby, Herbert, \& Depledge, 2013) a avaliação do serviço prestado é indicada pelas contribuições sociais de espaços urbanos verdes, reconhecidas e evidenciadas pelos efeitos positivos de bem-estar (Bragg, Wood, Barton, \& Pretty, 2015), facilmente percebidos. Segundo Tombs e McColl-Kennedy (2012), um benefício percebido, por exemplo, seria o valor na melhoria na qualidade de vida, pelo acesso ou restrição a um serviço, na inovação nos processos de prestação de um serviço, na gerencia de falhas do serviço, ou investigando os elementos de design de serviço.

\section{METODOLOGIA}

Este trabalho é composto por duas metodologias alinhadas para atingir o objetivo.

\section{$\mathbf{1}^{\mathrm{a}}$ fase metodológica}

A primeira é um levantamento teórico por meio do qual se baseiam as dimensões fundamentadas nos construtos propostos pela avaliação dos $7 \mathrm{ps}$ de marketing de serviço e pelas dimensões da qualidade, alinhadas no instrumento de pesquisa, demostradas no quadro 3. O caráter exploratório propõe a compreensão e a ampliação dos fatores que envolvem as dimensões propostas na literatura e as encontradas na coleta empírica. Uma vez que o objetivo proposto por este estudo é apontar os atributos que compõe a avaliação dos usuários, uma abordagem qualitativa do tipo exploratória seria adequada.

Promovendo a inserção do pesquisador na coleta de dados no ambiente proporcionaria a interpretação de um fenômeno e a atribuição de significados conforme defendido por Yin (2011). Da mesma forma Malhotra (2006) aconselha que a pesquisa exploratória seja justificada quando pretende-se ampliar os conhecimento de um determinado assunto.

Analisadas durante o processo de coleta a saturação foi encontrada ao se atingir 77 vistas totalizando 17 parques visitados na cidade de Belo Horizonte. A coleta dos dados foi realizada entre os meses de Abril e Maio de 2016, em dias e parques aleatórios por pesquisadores 
treinados para esta coleta. Assim, esta pesquisa foi realizada em 17 parques da cidade de Belo Horizonte, MG.

Tabela 1 - Parques visitados para avaliação da qualidade

\begin{tabular}{|c|c|}
\hline $\begin{array}{l}\text { Parque } \\
\text { Pesquisado }\end{array}$ & $\begin{array}{c}\text { Numero de } \\
\text { coletas }\end{array}$ \\
\hline 1. Parque Municipal Américo Renné Giannetti & 24 \\
\hline 2. Parque das Mangabeiras & 15 \\
\hline 3. Parque Ecológico da Pampulha & 9 \\
\hline 4. Parque Elias Michel Farah & 5 \\
\hline 5. Parque JK & 3 \\
\hline 6. Parque Ecológico Fazenda Lagoa do Nado & 3 \\
\hline 7. Parque Renato Azeredo & 3 \\
\hline 8. Parque Ecológico e Cultural Professor Marcos Mazzoni & 2 \\
\hline 9. Parque Ismael de Oliveira Fábregas & 2 \\
\hline 10. Parque Municipal Ursulina Andrade Mello & 2 \\
\hline 11. Parque Municipal Rosinha Cadar & 2 \\
\hline 12. Horto Municipal de Betim & 1 \\
\hline 13. Parque da Serra do Curral & 1 \\
\hline 14. Parque Ecológico Roberto Burle Marx & 1 \\
\hline 15. Parque Jornalista Eduardo Couri - Barragem Santa Lúcia & 1 \\
\hline 16. Parque Linear & 1 \\
\hline 17. Parque Universitário & 1 \\
\hline Total & 77 \\
\hline
\end{tabular}

Fonte: Dados da Pesquisa, 2016.

O roteiro semiestruturado foi desenvolvido com embasamento do referencial teórico e continha 27 perguntas (ver Quadro 3). Em média cada abordagem durava 8 minutos e eram gravadas com a autorização prévia do entrevistado.

Quadro 3 - Instrumento de pesquisa qualitativa

\begin{tabular}{|c|c|c|}
\hline Questionamento & Base teórica & Fonte \\
\hline $\begin{array}{l}\text { Qual sua relação com os parques da cidade? Fale um pouco de } \\
\text { seus interesses, motivações para visita, frequência de visitas. }\end{array}$ & $\begin{array}{l}\text { Perfil do usuário } \\
\text { Motivação da vista } \\
\text { Frequência }\end{array}$ & $\begin{array}{c}\text { Saatcioglu } \\
\text { e Ozanne } \\
(2013) \\
\text { Kim, Lee e } \\
\text { Klenosky (2003). }\end{array}$ \\
\hline Como um parque pode interferir na vida das pessoas? & \multirow[b]{2}{*}{ Benefícios } & \multirow{2}{*}{$\begin{array}{l}\text { Castilho, Dolbec e } \\
\text { Veresiu (2016) } \\
\text { Davis et al. (2016) }\end{array}$} \\
\hline $\begin{array}{l}\text { Quais as consequências de uma cidade sem parques? Quais as } \\
\text { consequências de uma vida sem lazer? }\end{array}$ & & \\
\hline $\begin{array}{l}\text { Você se lembra de alguma vista a um parque? Se sim poderia } \\
\text { narrá-la? (Com quem foi a esta visita? Como foi? Por que esta } \\
\text { vista está viva em sua memória?) }\end{array}$ & \multirow{3}{*}{ Emoções } & \multirow{3}{*}{ Oliver (1997) } \\
\hline Por que escolheu este parque? O que ele representa para você? & & \\
\hline $\begin{array}{c}\text { Quais as suas sensações quando está neste parque? } \\
\text { Você sente a mesma coisa em outros lugares? Que lugares? }\end{array}$ & & \\
\hline O que levaria você a não frequentar este parque? & \multirow{2}{*}{$\begin{array}{l}\text { Expectativas } \\
\text { (Item reverso) }\end{array}$} & \multirow{2}{*}{$\begin{array}{c}\text { Saatcioglu e } \\
\text { Ozanne (2013) }\end{array}$} \\
\hline O que te impede de visitar mais vezes este parque? & & \\
\hline $\begin{array}{l}\text { Nesta vista, você encontrou no parque o que esperava? Detalhe } \\
\text { sua resposta. }\end{array}$ & Expectativas & Sterry (2004) \\
\hline Que elementos deste parque te surpreenderam positivamente? & \multirow{2}{*}{$\begin{array}{l}\text { Tangíveis } \\
\text { Confiança }\end{array}$} & \multirow{2}{*}{$\begin{array}{l}\text { Fitzsimmons e } \\
\text { Fitzsimmons }\end{array}$} \\
\hline Que elementos deste parque te surpreenderam negativamente? & & \\
\hline
\end{tabular}




\begin{tabular}{|c|c|c|}
\hline & $\begin{array}{c}\text { Presteza/atendimento } \\
\text { Garantia/segurança } \\
\text { Empatia }\end{array}$ & $(2000)$ \\
\hline Como você descreveria essa visita que fez? & Experiência & $\begin{array}{l}\text { Vargo e Lusch } \\
\text { (2008) }\end{array}$ \\
\hline $\begin{array}{l}\text { Se tivesse que contar para alguém que visitou esse parque, o que } \\
\text { iria dizer? }\end{array}$ & \multirow{6}{*}{$\begin{array}{l}\text { Praça } \\
\text { Produto } \\
\text { Produto } \\
\text { Promoção } \\
\text { vidência física } \\
\text { Processos }\end{array}$} & \multirow{6}{*}{$\begin{array}{l}\text { Lovelock, Wirtz e } \\
\text { Hemz (2011) }\end{array}$} \\
\hline $\begin{array}{l}\text { Você concorda com investimentos governamentais em parques? } \\
\text { Estes investimentos deveriam prioritários? Por quê? }\end{array}$ & & \\
\hline $\begin{array}{l}\text { Que sugestões você daria para melhorar os aspectos negativos } \\
\text { que citou? }\end{array}$ & & \\
\hline $\begin{array}{l}\text { O que você diria das placas e sinalizações dentro do parque? E } \\
\text { em relação à limpeza? }\end{array}$ & & \\
\hline $\begin{array}{l}\text { O que você diz dos acessos, da manutenção e da segurança do } \\
\text { parque? }\end{array}$ & & \\
\hline $\begin{array}{l}\text { O que os funcionários de um parque fazem? Como você avalia a } \\
\text { presteza, a cordialidade e serviço prestado pelos funcionários? }\end{array}$ & & \\
\hline
\end{tabular}

Fonte: Instrumentalização da Pesquisa, 2016.

Todos os entrevistados eram usuários dos parques e a amostra encerrada pela saturação dados coletados por meio de questionários. Os 77 usuários convidados por conveniência, sem distinção apenas pela concordância em participar da entrevista. Foi necessário identificar o usuário e sua frequência para garantir que o mesmo possuísse conhecimento suficiente para avaliar certos elementos dos parques.

A caracterização da amostra de entrevistados foi necessária para garantir a diversidade de perfis, e por esta razão composta em termos de gênero: 30 homens e 47 mulheres, com idade entre as faixas de até 25; composta por 29 indivíduos, entre 26 e 35 anos; 16 indivíduos, entre 36 a 45 anos; 11 indivíduos, acima de 46 anos 18 pessoas. A predominância de escolaridade é de ensino médio completo, mas destacasse faixas como a de ensino fundamental ou ainda que não completaram este ciclo.

O tratamento da matriz de dados foi realizado por meio de análise de conteúdo, seguindo os procedimentos sistematizados e padronizados para descrever o conteúdo de mensagens (Bardin, 2011). A organização da análise seguiu os três polos cronológicos defendidos por Bardin (2011): 1) uma pré-análise; 2) a exploração do material; 3) a inferência e a interpretação. O agrupamento de análise temática busca-se desvendar núcleos de sentido por meio da frequência de termos que possam contribuir para as análises (Minayo, 2007).

Buscando contribuir para a amplitude de dimensões as analises quanto os resultados obtidos expressões e sentidos contrastados com as referências bibliográficas, buscaram-se conjuntamente com o objetivo. As categorias iniciais encontradas bem como a discussão e inferências são apresentados no próximo tópico. 


\begin{tabular}{|c|c|c|}
\hline Mensuração qualidade do serviço & Falas dos entrevistados & Base teórica \\
\hline $\begin{array}{c}\text { Dimensões da experiência: } \\
\text { Entretenimento, Educacional, } \\
\text { Escapista e Estética. } \\
\end{array}$ & \multirow[b]{2}{*}{$\begin{array}{l}\text { "só frequentava por conta de eventos esportivos ou } \\
\text { culturais atípicos." } \\
\text { "Os parques podem ser utilizados em um ambiente de } \\
\text { aprendizado quando fornecem a conscientização do } \\
\text { meio ambiente, da cultura, e dos meios sociais à } \\
\text { população. Como por exemplo, neste parque havia } \\
\text { um museu que explorava o folclore brasileiro; havia } \\
\text { uma biblioteca com um excelente acervo que ia desde } \\
\text { as crianças com livros infantis e gibis, aos adultos, } \\
\text { com livros mais complexos; havia uma atividade de } \\
\text { musica e uma oficina de massinha de modelar para as } \\
\text { crianças, e, descobri por meio da minha tia, que ali } \\
\text { todos os domingos tem rodas de músicas que } \\
\text { estimulam a musicalização nas crianças; há também } \\
\text { ali grupos da terceira idade como a academia da } \\
\text { cidade e grupos de tai-chi e yoga.” }\end{array}$} & $\begin{array}{c}\text { (Pine } \\
\& \\
\text { Gilmore, 2011) } \\
\end{array}$ \\
\hline $\begin{array}{c}\text { Significância do espaço: } \\
\text { Para o contexto turístico e de } \\
\text { conhecimento cultural, a novidade } \\
\text { promovem satisfação. }\end{array}$ & & $\begin{array}{l}\text { (Kim, Lee, \& } \\
\text { Klenosky, } \\
\text { 2003) }\end{array}$ \\
\hline $\begin{array}{l}\text { Convivência e o contato com outros } \\
\text { usuários: } \\
\text { Avaliados pela coletividade } \\
\text { (relacional e social) }\end{array}$ & $\begin{array}{c}\text { "Os parques são ótimas oportunidades de lazer } \\
\text { público e de socialização nas cidades. São lugares } \\
\text { onde todas as pessoas da cidade podem se encontrar e } \\
\text { conviver". } \\
\text { "No momento em que você vai a um parque } \\
\text { frequentemente, acaba por conhecer várias pessoas } \\
\text { que também vão ao parque com a mesma frequência, } \\
\text { como os seus vizinhos, e forma vínculos com essas } \\
\text { pessoas, depois deste novo relacionamento } \\
\text { estabelecido você começa a marcar encontros com } \\
\text { elas o que, de certa forma, muda a sua rotina e a } \\
\text { deles." }\end{array}$ & $\begin{array}{c}\text { (Verhoef, et } \\
a l ., 2009)\end{array}$ \\
\hline $\begin{array}{l}\text { A satisfação pode ser derivada de } \\
\text { sentimentos como: } \\
\text { Prazer, Alívio, Novidade, Excitação e } \\
\text { Surpresa do humor, alivio do estresse, } \\
\text { o relaxamento e reflexão, } \\
\text { desaceleração. }\end{array}$ & $\begin{array}{l}\text { “. Pessoas que vão para o parque para ter um } \\
\text { momento de reflexão e de descanso, seja depois do } \\
\text { trabalho ou durante uma pausa, com certeza } \\
\text { revigoram os seus ânimos. Indo mais além, acredito } \\
\text { que o parque possa até estreitar os laços de uma } \\
\text { família ou de um casal: quando a visita se torna uma } \\
\text { prática comum e eles tem ali um momento de prazer } \\
\text { e de intimidade de ideias.” }\end{array}$ & (Oliver, 1997) \\
\hline $\begin{array}{c}\text { Hedonismo: } \\
\text { Sendo bem-estar, qualidade de vida e } \\
\text { saúde as mais citadas. Nota-se que o } \\
\text { lazer, a recreação e pratica esportiva } \\
\text { está associado à prazer hedonismo, e } \\
\text { que a participação em eventos, a } \\
\text { reunião com amigos ou família esta } \\
\text { associada a emoção. }\end{array}$ & $\begin{array}{c}\text { “"Fiquei surpreendido com o parque ecológico da } \\
\text { pampulha. Já fazem doze anos desde sua inauguração } \\
\text { e nunca tive a oportunidade e interesse em visitá-lo. } \\
\text { Neste domingo, tive o prazer de conhecer um espaço } \\
\text { tranquilo, seguro e limpo para aproveitar junto com } \\
\text { minha família e amigos. " }\end{array}$ & $\begin{array}{l}\text { (Kim, Lee, } \& \\
\text { Klenosky, } \\
\text { 2003) }\end{array}$ \\
\hline $\begin{array}{l}\text { Aspectos tangíveis: } \\
\text { Caracteristicas concretas que são } \\
\text { capazes de capturar satisfação e } \\
\text { avaliação da qualidade }\end{array}$ & $\begin{array}{c}\text { "Imagino que as pessoas iriam buscar outras formas } \\
\text { alternativas de lazer, mas infelizmente, muitas delas } \\
\text { seriam privadas e não gratuitas. } \\
\text { "Os parque gratuitos porque são alternativas de } \\
\text { programas para quando as pessoas buscam algo } \\
\text { divertido e barato. } \\
\text { "Um parque possui inúmeras funções, entre elas a } \\
\text { estética, ecológica e de lazer. Na minha opinião, as } \\
\text { pessoas desejam encontrar nos parques eventos } \\
\text { culturais, atividades recreativas e opções de lazer } \\
\text { alternativas. }\end{array}$ & $\begin{array}{l}\text { (Fitzsimmons } \\
\quad \& \\
\text { Fitzsimmons, } \\
\text { 2000) }\end{array}$ \\
\hline & $\begin{array}{l}\text { "Em relação a sinalização, eu diria que poderia } \\
\text { melhorar mas ainda assim é efetiva, até certo ponto. }\end{array}$ & $\begin{array}{l}\text { (Lovelock, } \\
\text { Wirtz, \& }\end{array}$ \\
\hline
\end{tabular}




\begin{tabular}{|l|c|c|}
\hline & $\begin{array}{c}\text { A limpeza é um dos pontos fracos do parque, apesar } \\
\text { das várias lixeiras espalhadas por ele. } \\
\text { "É muito bem sinalizado, com placas, mapas, etc. A } \\
\text { limpeza é feita diariamente aparentemente, mas ainda } \\
\text { sim se encontra sujeira. } \\
\text { "O parque é acessível para deficientes físicos com } \\
\text { corrimões e rampas com inclinação leve. Embora } \\
\text { para cegos não seja tão cessível. Manutenção muito } \\
\text { boa do parque com parcerias inclusive com o Japão } \\
\text { no jardim de cerejeiras. Segurança visível com os } \\
\text { funcionários e polícia que rondava o local." }\end{array}$ \\
\hline
\end{tabular}

Fonte: Instrumentalização da Pesquisa, 2016.

Destaca-se a incidência de "bem-estar" como relacionada a mediação de avaliação de qualidade, presente no relato dos entrevistados constantemente vinculada com a saúde física se destaca, mas o componente saúde mental, a interatividade e interação social (Bragg, Wood, Barton, \& Pretty, 2015), relaxamento e desestresse (White, et al., 2013) caracterizam que saúde mental são descritos. Ou seja, serviços disponibilizados a uma população geram efeitos positivos relacionados à saúde física e saúde mental de seus habitantes (Davis, Ozanne, \& Hill, 2016).

Outra observação que merece destaque é vinculada ao aprendizado e a educação, segundo Pine e Gilmore (2011), o componente educacional, e cognitivo promovem respostas comportamentais proporcionados por uma relação com um estímulo (Brakus, Schmitt, \& Zarantonello, 2009) e referenciada nas entrevistas em situações que repetidamente se envolve com "crianças, passeios com filhos", proporcionam uma serie de situações de aprendizado e desenvolvimento cognitivo e social, extrapolando a importância de parques verdes como um espaço de conhecimento para de construção social com e pelos usuários.

Os estudos de Pierskalla, Lee, Stein, Anderson e Nickerson (2004) já incluíam nas recomendações de estudos futuros a especial atenção de variáveis como: condições locais que afetam a qualidade do serviço. Observa-se que as instalações em um parque são muitas vezes específicas de um determinado espaço, o que diferencia um parque do outro (Crilley, Weber, \& Taplin, 2012). Há diferenças em quais os esportes são oferecidos, na estrutura e na metragem do parque. Ou seja, cada Parque é único, alguns se diferenciam ao oferecer experiências diversificadas que vão ao encontro dos seus públicos, uns oferecendo serviços sociais, outros culturais ou educacionais.

Considerados então como áreas de múltiplos usos, a avaliação da qualidade deste equipamento público é limitada, o grau em que descobertas quando associadas com a qualidade do serviço não podem ser generalizadas (Sempik \& Bragg, 2013). Este estudo considerou esta afirmação. 
Assim, como já afirmado por Edginton e Chen (2014) experiências vividas pelo usuário frente a estímulos, frente aos serviços e frente as suas expectativas podem hipoteticamente estarem relacionadas a entrega dos benefícios, em um contexto específico. Investigar a qualidade para além, para a experiência e o pós-experiencial tendo como compromisso de melhorar a experiência, também é uma recomendação.

Os parques se relacionam com o aprendizado de seus visitantes, da historia da cidade, da vegetação nativa da preservação. A comunidade neste ambiente relata que é possível aprender compartilhando a responsabilidade de cuidar do local, das arvores. Assim a oportunidade de aprender e contribuir para aspectos da cidadania derivadas de valores pessoais, cumprindo seu papel educacional. Outra possibilidade de aprendizado e educação seria a inserção cultural, a utilização destes espaços para eventos de teatro, musica e atividades, shows diversos.

Quadro 5 - Categorias e dimensões

\begin{tabular}{|c|c|c|}
\hline $\begin{array}{c}\text { CÓDIGOS } \\
\text { (Codificação aberta) }\end{array}$ & $\begin{array}{c}\text { CATEGORIAS } \\
\text { (Codificação Axial) }\end{array}$ & DIMENSÕES \\
\hline $\begin{array}{l}\text { Estrutura } \\
\text { Áreas de lazer } \\
\text { Quadras esportivas } \\
\text { Vandalismo e Pichações } \\
\text { Lixo e Lixeiras } \\
\text { Conservação e Limpeza } \\
\text { Gramado verde }\end{array}$ & $\begin{array}{l}\text { ASPECTOS } \\
\text { TANGÍVEIS }\end{array}$ & $\begin{array}{l}\text { 1. O parque é bem organizado. } \\
\text { 2. Os equipamentos para prática de esportes estão } \\
\text { funcionando. } \\
\text { 3. parque está limpo. } \\
\text { 4. Os bancos são confortáveis. } \\
\text { 5. Os brinquedos são conservados. } \\
\text { 6. As áreas verdes são bem cuidadas e } \\
\text { preservadas. }\end{array}$ \\
\hline $\begin{array}{l}\text { Distribuição de parques } \\
\text { pela cidade } \\
\text { Proximidade da } \\
\text { residência } \\
\text { Deslocamento e } \\
\text { transporte } \\
\text { Divulgação } \\
\text { Acesso a informação }\end{array}$ & ACESSO & $\begin{array}{l}\text { 7. O parque é bem localizado. } \\
\text { 8. É fácil chegar a esse parque. } \\
\text { 9. horário de funcionamento do parque é } \\
\text { adequado. } \\
\text { 10. Há boa divulgação das atividades que } \\
\text { acontecem nesse parque. }\end{array}$ \\
\hline $\begin{array}{l}\text { Sinalização } \\
\text { Placas e mapas } \\
\text { indicativos } \\
\text { Facilidade de acesso } \\
\text { interno } \\
\text { Informação e guias } \\
\text { Acessibilidade }\end{array}$ & $\begin{array}{l}\text { ACESSIBILIDADE } \\
\text { INTERNA }\end{array}$ & $\begin{array}{l}\text { 11. É fácil se orientar pelas placas e pela } \\
\text { sinalização internas deste parque. } \\
\text { 12. O piso facilita as caminhadas pelo parque. } \\
\text { 13. Existe preocupação em facilitar o acesso a } \\
\text { pessoas que têm mobilidade reduzida. }\end{array}$ \\
\hline $\begin{array}{l}\text { Vigilância e } \\
\text { Policiamento } \\
\text { Controle fluxo entrada } \\
\text { Risco doenças }\end{array}$ & SEGURANÇA & $\begin{array}{l}\text { 14. O parque possui vigilância ou policiamento. } \\
\text { 15. Todos os ambientes do parque são seguros . } \\
\text { 16. Parece seguro em todos os horários. }\end{array}$ \\
\hline $\begin{array}{l}\text { Beleza visual } \\
\text { Espaço de preservação } \\
\text { Contemplação } \\
\text { Elemento estético } \\
\text { Elemento paisagístico } \\
\text { Ponto de referencia }\end{array}$ & ESTÉTICA & $\begin{array}{l}\text { 17. Este parque é bonito. } \\
\text { 18. Este parque embeleza a cidade. } \\
\text { 19. Você gosta de tirar fotos neste parque. }\end{array}$ \\
\hline
\end{tabular}




\begin{tabular}{|c|c|c|}
\hline $\begin{array}{l}\text { Quiosque de alimentos e } \\
\text { lanchonetes } \\
\text { Bancos e área de } \\
\text { descanso } \\
\text { Bebedouros } \\
\text { Sanitários }\end{array}$ & $\begin{array}{l}\text { CONFORTO AO } \\
\text { USUÁRIO }\end{array}$ & $\begin{array}{l}\text { 20. O parque possui atrativos para todas as idades. } \\
\text { 21. O parque oferece vasta gama de possibilidades } \\
\text { para que as pessoas dele usufruam. } \\
\text { 22. Parque possui mesas suficientes que podem ser } \\
\text { 23. Asadas para estudar ou para fazer um lanche. } \\
\text { bebidas são apropriadas. }\end{array}$ \\
\hline $\begin{array}{l}\text { Número de pessoas } \\
\text { usando o espaço } \\
\text { A promoção de eventos } \\
\text { integradores } \\
\text { Momentos em famíla } \\
\text { Integração entre as } \\
\text { pessoas } \\
\text { Confraternização entre } \\
\text { amigos }\end{array}$ & SOCIABILIDADE & $\begin{array}{l}\text { 24. O parque é frequentado por pessoas de } \\
\text { diferentes classes sociais. } \\
\text { 25. Os espaços de integração entre as pessoas são } \\
\text { adequados. } \\
\text { 26. parque tem capacidade de receber eventos. } \\
\text { 27. O parque favorece a inclusão social. }\end{array}$ \\
\hline
\end{tabular}

Fonte: Instrumentalização da Pesquisa, 2016.

Os entrevistados consideram os parques como uma opção alternativa de lazer (Bragg, Wood, \& Barton, 2013). As opções de shoppings, baladas e a justificativa que amigos não vão a parques está presente nas respostas. As cognições e respostas comportamentais proporcionados pela relação com um estímulo (Brakus, Schmitt, \& Zarantonello, 2009) estão presentes e por esta razão podem ser capturadas por uma abordagem quantitativa.

Apresentado o método de pesquisa de como foi constituído, o instrumento survey de pesquisa foi aplicado. Os resultados apresentados são alcançados por identificar variáveis que extrapolam as propostas teóricas existentes, ampliam a construção inicial do modelo, possibilitando o teste de hipóteses testadas.

\section{$2^{\text {a }}$ fase metodológica}

A segunda fase caracterizada por um estudo do tipo survey, que atingiu 737 questionários em seu total, sendo que foram considerados validos após purificação da matriz de dados que retirou outilieres variados, univariados, dados extremos e ausentes. Os resultados obtidos permitiram concluir que a qualidade percebida pelos consumidores com relação aos serviços prestados é um construto multidimensional.

Uma breve descrição do perfil da amostra destaca que o número de mulheres (62\%) foi bastante superior ao de homens (36\%), a busca por uma amostra proporcional evitaria qualquer tipo de distorção, em que tenha nas opiniões femininas, um peso maior do que consideradas as opiniões masculinas (em proporcionalidade). Duas justificativas podem ser apresentadas para essa desproporção. Alguns entrevistadores relataram que além da disponibilidade e interesse das mulheres em responder o questionário, o número de mulheres era visivelmente maior, em proporcionalidade ao número de frequentadores do parque, em grupos ou acompanhadas de crianças. 
A predominância de um perfil de respondentes feminino na amostra, prevalece no estrato que corresponde à idade de 20 a 26 anos (37\%), com escolaridade ensino médio (61\%) e ainda com rendimentos familiares de até 2 salários mínimos (29\%). Este perfil combinado representa a maioria dos visitantes, de baixa renda, atraído pelo lazer gratuito e acessível a estas classes, já evidenciados na literatura uma relação direta entre renda e escolaridade para a escolha de uma opção de lazer.

O survey possui uma escala de 0 a 10 , indo de "discordo totalmente" até "concordo totalmente", assim a operacionalização sintética das variáveis escalares que compõem o instrumento são construídas em escala Likertt. Base esta, que será necessária para utilizarmos nas analise multivariadas subsequentes.

\subsection{Análise fatorial exploratória}

Com o intuito de maximizar o poder explicativo de um conjunto de variáveis, Hair, Wolfinbarger, Money, Samouel e Page (2015) recomendam que a técnica ao considerar todos os itens. simultaneamente confirma a adequação da escala.

Sendo que pôde-se observar que o teste de Esfericidade de Bartlett demonstra a existência de relações significativas entre os itens e testa a hipótese nula de que não há correlação significativa entre os itens, sendo, portanto, a matriz de correlação igual à matriz identidade. O nível de significância do Teste TBM ser inferior a $1 \%$.

Quadro 6 - Agrupamento fatorial

\begin{tabular}{|c|c|c|c|c|c|c|c|c|}
\hline & Itens & \begin{tabular}{l|} 
Carga \\
Fatorial
\end{tabular} & $\begin{array}{l}\text { Comuna } \\
\text { lidade }\end{array}$ & Alpha & Itens & & & \\
\hline V1 & AASPTANG1 & 0,667 & 0,730 & \multirow{6}{*}{867} & ... o parque é bem organizado. & AAT1 & \multirow{6}{*}{ ATT } & \multirow{6}{*}{$\begin{array}{l}\text { Avaliação } \\
\text { Aspectos } \\
\text { Tangíveis }\end{array}$} \\
\hline $\mathrm{V} 2$ & AASPTANG2 & 0,687 & 0,640 & & $\begin{array}{l}\text {... os equipamentos para prática de } \\
\text { esportes parecem adequados. }\end{array}$ & AAT2 & & \\
\hline V3 & AASPTANG3 & 0,525 & 0,656 & & ... o parque está limpo. & AAT3 & & \\
\hline V4 & AASPTANG4 & 0,605 & 0,674 & & ... os bancos são confortáveis. & AAT4 & & \\
\hline V5 & AASPTANG5 & 0,504 & 0,544 & & ... os brinquedos são conservados. & AAT5 & & \\
\hline V6 & AASPTANG6 & 0,572 & 0,668 & & $\begin{array}{l}\ldots \text { as áreas verdes são bem } \\
\text { cuidadas e preservadas. }\end{array}$ & AAT6 & & \\
\hline V7 & AACESSO1 & 0,677 & 0,651 & \multirow{3}{*}{.740} & ... o parque é bem localizado. & AFA1 & \multirow{3}{*}{ AFA } & \multirow{3}{*}{$\begin{array}{l}\text { Avaliações } \\
\text { Facilidade } \\
\text { de Acesso }\end{array}$} \\
\hline V8 & AACESSO 2 & 0,739 & 0,680 & & ... é fácil chegar a esse parque. & AFA2 & & \\
\hline V9 & AACESSO3 & 0,708 & 0,638 & & $\begin{array}{l}\text {... o horário de funcionamento do } \\
\text { parque é adequado. }\end{array}$ & AFA3 & & \\
\hline V10 & AACESSO4 & 0,787 & 0,715 & \multirow{4}{*}{.862} & $\begin{array}{l}\text {... há boa divulgação das } \\
\text { atividades que acontecem nesse } \\
\text { parque. }\end{array}$ & AS1 & \multirow{4}{*}{ AS } & \multirow{4}{*}{$\begin{array}{l}\text { Avaliação } \\
\text { Segurança }\end{array}$} \\
\hline V11 & AACESIBIT1 & 0,827 & 0,739 & & $\begin{array}{l}\text {... é fácil se orientar pelas placas e } \\
\text { pela sinalização internas deste } \\
\text { parque. }\end{array}$ & AS2 & & \\
\hline V12 & AACESIBIT2 & 0,700 & 0,651 & & $\begin{array}{l}\text {... o piso facilita as caminhadas } \\
\text { pelo parque. }\end{array}$ & AS3 & & \\
\hline V13 & AACESIBIT3 & 0,782 & 0,737 & & ... existe preocupação em facilitar & AS4 & & \\
\hline
\end{tabular}




\begin{tabular}{|c|c|c|c|c|c|c|c|c|}
\hline & & & & & $\begin{array}{l}\text { o acesso a pessoas que têm } \\
\text { mobilidade reduzida. }\end{array}$ & & & \\
\hline V14 & ASEGURA1 & 0,722 & 0,671 & & $\begin{array}{l}\text {... o parque possui vigilância ou } \\
\text { policiamento. }\end{array}$ & AS5 & & \\
\hline V15 & ASEGURA2 & 0,735 & 0,660 & & $\begin{array}{l}\text {... todos os ambientes do parque } \\
\text { são seguros. }\end{array}$ & AS6 & & \\
\hline V16 & ASEGURA3 & 0,684 & 0,633 & & $\begin{array}{l}\text {... parece seguro em todos os } \\
\text { horários. }\end{array}$ & AS7 & & \\
\hline V17 & AESTETIC1 & 0,551 & 0,641 & \multirow{4}{*}{, 822} & ... o parque é bonito. & $\mathrm{AB} 1$ & \multirow{4}{*}{$\mathrm{AB}$} & \multirow{4}{*}{$\begin{array}{l}\text { Avaliação } \\
\text { Beleza }\end{array}$} \\
\hline V18 & AESTETIC2 & 0,546 & 0,659 & & ... o parque embeleza a cidade. & $\mathrm{AB} 2$ & & \\
\hline V19 & AESTETIC3 & 0,682 & 0,737 & & ... o parque é ideal para tirar fotos. & $\mathrm{AB} 3$ & & \\
\hline V20 & ACONFOR1 & 0,712 & 0,741 & & $\begin{array}{l}\text {... o parque possui atrativos para } \\
\text { todas as idades. }\end{array}$ & $\mathrm{AB} 4$ & & \\
\hline V23 & ACONFOR4 & 0,706 & 0,753 & \multirow{5}{*}{, 809} & $\begin{array}{l}\text {... as opções para o consumo de } \\
\text { bebidas e comidas são } \\
\text { apropriadas. }\end{array}$ & API1 & \multirow{5}{*}{ API } & \multirow{5}{*}{$\begin{array}{l}\text { Avaliação } \\
\text { Propensão } \\
\text { a } \\
\text { Integração }\end{array}$} \\
\hline V24 & ASOCIABIL1 & 0,588 & 0,688 & & $\begin{array}{l}\text {... o parque é frequentado por } \\
\text { pessoas de diferentes classes } \\
\text { sociais. }\end{array}$ & API2 & & \\
\hline V25 & ASOCIABIL2 & 0,583 & 0,689 & & $\begin{array}{l}\text {... os espaços de integração entre } \\
\text { as pessoas são adequados. }\end{array}$ & API3 & & \\
\hline V26 & ASOCIABIL3 & 0,607 & 0,649 & & $\begin{array}{l}\text {... o parque tem boa capacidade de } \\
\text { receber grandes eventos. }\end{array}$ & API4 & & \\
\hline V27 & ASOCIABIL4 & 0,637 & 0,643 & & $\begin{array}{l}\text {... o parque favorece a inclusão } \\
\text { social. }\end{array}$ & API5 & & \\
\hline
\end{tabular}

Fonte: Instrumentalização da Pesquisa, 2016.

A Avaliação da qualidade (QUA) construto testado quanto à existência de subfatores (e também dimensões) refletindo a qualidade percebida foi a priori definido pela existência de 7 fatores adjacentes. Porém a solução fatorial apresentou uma redução dos fatores iniciais para 5 fatores, em uma proposta de iniciais 27 itens ou variáveis, para 25. Uma extração de dois itens é considerada pequena comparada ao volume de variáveis testadas, em função da baixa carga fatorial os itens ACONFOR2 (V21. "O parque oferece vasta gama de possibilidades para que as pessoas dele usufruam”) e ACONFOR3 (V22 - “... Parque possui mesas suficientes que podem ser usadas para estudar ou para fazer um lanche") uma vez que apresentaram carga fatorial abaixo de 0,5. O critério de corte destes fatores se baseia em Kline (2011) que afirma que a representação da correlação da variável com seu respectivo Dimensão (ou fator) quando $>0,50$ que possuiria uma "significância prática".

Os Itens ACONFOR1 (... o parque possui atrativos para todas as idades) foi deslocado para a dimensão Estética e renomeada para AVALIAÇÂO BELEZA, composta agora por 4 variáveis. O deslocamento deste item por meio de uma análise semântica faz sentido, uma vez que a palavra "atrativos" pode ter inferido um conceito próximo de atraente e relacionado a beleza. Já ACONFOR4 (... as opções para o consumo de bebidas e comidas são apropriadas foi deslocado para a dimensão Sociabilidade e renomeada para AVALIAÇÂO PROPENÇÂO A INTEGRAÇÃO. As justificativas dos deslocamentos são suportadas, também por meio de 
uma inferência semântica, dos itens, em que o respondente pode ter associado bebidas e comidas como uma variável, mas fortemente associada a integração ou ao convívio e a um momento de trocas sociais.

Os agrupamentos demostrado no Quadro 5 portanto destacam que das 7 dimensões iniciais a ocorrência do agrupamento dos fatores relacionados ao Conforto ao usuário foram distribuídos entre os dois itens da dimensão e ainda duas dimensões ACESSIBILIDADE e SEGURANÇA foram agrupadas em apenas uma. Tornando-se a dimensão com mais itens percebe-se que (1) o item “... há boa divulgação das atividades que acontecem nesse parque.” Relaciona-se com uma característica de que o usuário percebe a informação como um fator que gera segurança; (2) que os itens relacionados anteriormente com ACESSIBILIDADE também são percebidos como fatores que avaliam segurança.

O agrupamento dos itens por meio da AFE possibilitou a redução de duas dimensões, na verdade o agrupamento por fator. O restante das variáveis está com valores de comunalidade e de carga fatorial dentro do recomendado pela literatura, o que assegura a solução fatorial apresentada.

\section{CONSIDERAÇÕES FINAIS}

Este estudo atinge o objetivo proposto, a metodologia descrita se mostrou suficiente para que fosse possível compreender a decisão por uma rotulagem que distinga das dimensões estabelecidas, mas ainda assim mantendo-se ainda apropriada a literatura.

Assim as dimensões de QUA confirmam um construto multidimensional e também complexo. Sendo possível verificar por meio da AFE que (1) as dimensões das escalas anteriores utilizadas pela literatura podem ser refinadas e adaptadas; (2) que as maiores cargas estão em aspectos tangíveis da avaliação da qualidade de áreas verdes publicas; (3) a dimensão CONFORTO é medida por meio de outras dimensões.

A coleta de dados foi rica e possui informações que extrapolam as apresentadas neste artigo. Provavelmente a mesma base de dados pode ser reutilizada desmembrando as impressões dos parques individualmente. O que contribuirá para a construção do modelo apresentado e sua confirmação em módulos individuais. Através da matriz de dados organizada, é possível criar os itens de um questionário, pode-se utiliza-la para derivar pesquisas de profundidade, já que este estudo avançará, como parte integrante das pesquisas realizadas no grupo de pesquisa onde o modelo apresentado será testado. 
Este estudo apresenta algumas limitações, por se tratar de um estudo exploratório, ainda são necessários avanços em relação a abordagem e a mensuração do tema, inclusive, para encontrar e acrescentar variáveis não mencionadas no modelo proposto. O modelo não é exaustivo. Como a amostra é não representativa, selecionada por conveniência, um viés na percepção de estudantes apresenta a realidade de forma parcial.

Embasar decisões dos envolvidos na gestão de parques urbanos, como por exemplo, oferecer algo que anteriormente não estava à disposição do usuário, incrementa a oferta do prestador de serviço em atender as necessidades do consumidor. Pontua-se que embora seja preciso estar atenta a maior satisfação dos clientes ou da amplitude de oferecer o serviço a mais clientes não se pode esquecer de que o cliente é parte essencial na criação de valor do serviço e das inovações devem então ser feitas tendo como base nas necessidades do consumidor, seu desenvolvimento tende a apresentar resultados positivos na criação de valor.

\section{REFERÊNCIAS}

Akaka, M. A., Corsaro, D., Kelleher, C., Maglio, P. P., Seo, Y., Lusch, R. F., \& Vargo, S. L. (2014). The role of symbols in value cocreation. Marketing Theory, 14(3), 311-326.

Augustyn, M. M. \& Seakhoa-King, A. (2004). Is the servqual scale an adequate measure of quality in leisure, tourism and hospitality. In Advances in hospitality and leisure. Emerald Group Publishing Limited, 3-24.

Bardin, L. (2011). Análise de conteúdo. (4a. ed.). Lisboa: Edições 70.

Barra, O. A., Silva, S. A. P. S., Miranda, M. L. J., \& Vitorino, M. R. (2014). Avaliação de políticas públicas para o esporte. In V Congresso Sudeste de Ciências do Esporte.

Bettencourt, L. A., Lusch, R. L., \& Vargo, S. L. (2014). A service lens on value creation: marketing's role in achieving strategic advantage. California Management Review, 57(1), 4466.

Bitner, M. J. (1992). Servicescapes: The Impact of Physical Surroundings on Customers and Employees. Journal of Marketing, 56(2), 57-71

Bitner, M. J. \& Zeithaml, V. (2003). Marketing de serviços: a empresa com foco no cliente. (2a. ed.). Porto Alegre: Bookman.

Blackwell, R. D., Miniard, P. W., \& Engel, J. F. (2013). Comportamento do consumidor. São Paulo: Cengage Learning.

Bragg R., Wood C., \& Barton J. (2013). Ecominds effects on mental wellbeing: An evaluation for Mind. Colchester: University of Essex.

Bragg, R., Wood, C., Barton, J., \& Pretty, J. (2015). Wellbeing benefits from natural environments rich in wildlife: A literature review for the wildlife trusts. University of Essex. 
Brakus, J. J., Schmitt, B. H., \& L. Zarantonello. (2009). Brand experience: what is it? How is it measured? Does it affect loyalty?. Journal of Marketing, 73, 52-68.

Castilhos, R., Pierre-Yan D., \& Veresiu E. (2016). Introducing a Spatial Perspective to Analyze Market Dynamics. Marketing Theory, (forthcoming).

Chang, K. C. (2016). Effect of servicescape on customer behavioral intentions: Moderating roles of service climate and employee engagement. International Journal of Hospitality Management, 53, 116-128.

Christopher, M., Payne, A.F.T., \& Ballantyne, D. (1991). Relationship Marketing: Bringing Quality, Customer Service and Marketing Together. Butterworth Heinemann/CIM: Oxford.

Costa, S., Álvares, L., Maciel, M., Teixeira, M. C., Coimbra, V., Simão, K., Perna, S., \& Godinho, L. (2009). Os Espaços Livres na Paisagem de Belo Horizonte. Paisagem e Ambiente, (26), 51-72.

Crilley, G., Weber, D., \& Taplin, R. (2012). Predicting Visitor Satisfaction in Parks: Comparing the Value of Personal Benefit Attainment and Service Levels in Kakadu National Park, Australia. Visitor Studies, 15(2), 217-237.

Davis B. Ozanne, J., \& Hill, R. P. (2016). The Transformative Consumer Research Movement. Journal of Public Policy \& Marketing, 35(2), 159-169.

Edginton, C. R. \& Chen, P. (2014). Leisure as transformation. Sagamore Publishing, L.L.C.

Fitzsimmons, J. \& Fitzsimmons, M. (2000). Administração de Serviços: operações, estratégia e tecnologia de informação. Porto Alegre: Ed. Bookman.

Gronroos, C. (2009). Marketing: gerenciamento e serviços. Rio de Janeiro: Elsevier.

Hair Jr, J. F., Wolfinbarger, M., Money, A. H., Samouel, P., \& Page, M. J. (2015). Essentials of business research methods. Routledge.

Hoffman, K. D. \& Bateson, J. E. G. (2003). Princípios de Marketing de Serviços - Conceitos, Estratégias e Casos. São Paulo: Pioneira Thomson Learning.

Kaplan, R. S. \& Norton, D. P. (1997). A estratégia em ação: balanced scorecard. Rio de Janeiro: Campus.

Kim, S. S., Lee, C. K., \& Klenosky, D. B. (2003). The influence of push and pull factors at Korean national parks. Tourism Management, 24(2), 169-180.

Kline, R.B. (2011). Principles and Practice of Structural Equation Modeling. New York: Guilford Press.

Lindon, D., Lendrevie, J., Lévy, J., Dionísio, P., \& Rodrigues, J. V. (2013). Mercator XXI Teoria e prática do Marketing. Alfragide: Publicações Dom Quixote.

Lovelock, C., Wirtz, J., \& Hemzo, M. A. (2011). Marketing de Serviços: pessoas, tecnologia e estratégia. São Paulo: Pearson Prentice Hall.

Malhotra, N. (2006). Pesquisa de marketing: uma orientação aplicada. (4a. ed.). Porto Alegre: Bookman. 
Minayo, M. C. S. (2007). O desafio do conhecimento. Pesquisa qualitativa em saúde. São Paulo: HUCITEC.

Oliver, L. A. (1997). Satisfaction: a behavorial perspective on the consumer. Boston: Irwin/McGraw-Hill.

Ostrom, A. L., Parasuraman, A., Bowen, D. E., Patricio, L., \& Voss, C. A. (2015). Service research priorities in a rapidly changing contexto. Journal of Service Research, 18, 127-159.

Parasuraman, A., Zeithaml, V. A., \& Berry, L. (1985). A Conceptual Model of Service Quality and Its Implications for Future Research. Journal of Marketing, 49(4), 41-50.

Parasuraman, A., Zeithaml, V. A., \& Berry, L. (1988). SERVQUAL: A multiple- Item Scale for measuring consumer perceptions of service quality. Journal of Retailing, 64(1).

Pereira, M. T., Gimenes, M. L., da Silva, F. F., \& Zanatta, O. A. (2011). Desenvolvimento de indicador de qualidade de áreas verdes urbanas (IQAVU) e aplicação em cidades paranaenses. Revista em Agronegócio e Meio Ambiente, 5(1).

Pierskalla, C., Lee, M., Stein, T., Anderson, D., \& Nickerson, R. (2004). Understanding relationships among recreation opportunities: A meta-analysis of nine studies. Leisure Sciences, 26, 163-180.

Pine II, B. J. \& Gilmore, J. H. (2011). The Experience Economy. Boston: Harvard Business Review Press.

Prahalad, C. K. \& Ramaswany, V. (2004). The Future of Competition: Co-Creating Unique Value with Customers. Boston: Harvard Business School Press.

Saatcioglu, B. \& Ozanne J. L. (2013). A Critical Spatial Approach to Marketplace Exclusion and Inclusion. Journal of Public Policy \& Marketing, 32, 32-37.

Sempik, J. \& Bragg, R. (2013). Green Care: origins and activities. In Gallis C. (Ed.). Green Care: for Human Therapy, Social innovation, Rural economy, and Education. Nova Science Publishers, New York.

Sterry, P. (2004). An insight into the dynamics of family group visitors to cultural tourism destinations: Initiating the research agenda. In Smith, K.A. \& Schott, C. Proceedings of the New Zealand tourism and hospitality research conference 2004. Wellington, 298-406.

Tombs, A. G. \& McColl-Kennedy, J.R. (2013). Third party customers infecting other customers for better or for worse. Psychology and Marketing, 30(3), 277-292.

Vargo, S. L. \& Lusch, R. F. (2004). Evolving to a New Dominant Logic for Marketing. Journal of Marketing, 68, 1-17.

Vargo, S. L. \& Lusch, R. (2008). Service-dominant logic: Continuing the evolution. Journal of the Academy of Marketing Science, (1), 1-10.

Vargo, S. L. \& Lusch, R. F. (2016). Institutions and axioms: an extension and update of service-dominant logic. Journal of the Academy of Marketing Science, 44(1), 5-23.

Verhoef C. P., Lemon N. K., Parasuraman A., Roggeveen A., Tsiros M., \& Schlesinger A. L. (2009). Customer Experience Creation: Determinants, Dynamics and Management Strategies. Journal of Retailing, 85(1), 31-34. 
Vignati, F. (2008). Gestão de Destinos Turísticos: como atrair pessoas para pólos, cidades e países. Rio de Janeiro: SENAC.

Wendel, H. E. W., Zargerb, R. K., \& Mihelcic, J. R. (2012). Accessibility and usability: Green space preferences, perceptions, and barriers in a rapidly urbanizing city in Latin America. Landscape and Urban Planning, 107, 272-282.

White, M. P., Pahl, S., Ashbullby, K., Herbert, S., \& Depledge, M. H. (2013). Feelings of restoration from recent nature visits. Journal of Environmental Psychology, 35, 40-51.

Wieland, H., Polese, F., Vargo, S. L., \& Lusch, R. F. (2012). Toward a service (eco) systems perspective on value creation. International. Journal of Service Science, Management, Engineering, and Technology, 3(3), 12-25.

Yin, R. K. (2001). Estudo de caso: planejamento e métodos. (2a. ed.). Porto Alegre: Bookman.

Zeithaml, V. A. \& Berry, L. L. (1988). Servqual: A Multiple-Item Scale for Measuring Consumer Perceptions of Service Quality. Journal of Retailing, 64(1), 12-40.

Zeithaml, V. A., Parasuraman, A., \& Berry, L. L. (1990). Delivering Quality Service: balancing customer perceptions and expectations. New York: The Free Press. de parques públicos verdes urbanos. Revista de Turismo Contemporâneo, 7(2), 279-302. https://doi.org/10.21680/2357-8211.2019v7n2ID18199 